\title{
The Simian Virus 40 Large T Antigen Does Not Inhibit Translation of the 14-kDa Myelin Basic Protein mRNA in Reticulocyte Lysates or in Transfected Cells
}

\author{
$* \dagger$ S. Ueno, ${ }^{*}$ L. Foster, ${ }^{*}$ G. T. Hifumi, $\ddagger$ G. I. Tennekoon, and $*$ A. T. Campagnoni \\ *Developmental Biology Group, Mental Retardation Research Center, UCLA School of Medicine, Los Angeles, California; \\ $\ddagger$ Department of Pediatrics, University of Michigan Medical School, Ann Arbor, Michigan, U.S.A.; and \\ $\dagger$ Department of Neuropsychiatry, Ehime University School of Medicine, Ehime, Japan
}

\begin{abstract}
Viral $T$ antigens are transcription factors that have been suspected of inhibiting expression of the myelin basic protein (MBP) mRNA at the translational level in vitro and in vivo. The effect of simian virus 40 (SV40) large T antigen (T-ag) was examined on the translation of the 14-kDa MBP mRNA in reticulocyte lysates and on MBP expression after transfection into cells that express SV40 T-ag. SV40 T-ag did not inhibit translation of 14-kDa MBP cRNAs in cell-free translations even at $30 \mu \mathrm{M}$ $(\sim 600 \mu \mathrm{g} / \mathrm{ml}) \mathrm{T}$-ag. Permanent transfection of COS-1 cells (which endogenously express SV40 T-ag) with the 14-kDa MBP cDNA resulted in the expression of the 14-kDa MBP as determined by western blot analysis. Permanent transfection of N20.1 cells, an oligodendrocyte line immortalized with a temperaturesensitive SV40 T-ag, with the 14-kDa MBP cDNA construct also resulted in the expression of the 14-kDa MBP under conditions in which the cells expressed functional SV4O T-ag. These results indicate that SV40 T-ag does not prevent expression of the MBP gene at the translational level and that in those immortalized oligodendrocyte lines that express MBP mRNA, but not MBP protein, some factor other than the SV40 large T-ag is responsible for the posttranscriptional regulation. Key Words: Myelin basic protein mRNA-Simian virus 40 large $T$ antigen-Posttranscriptional regulation.

J. Neurochem. 64, 928-931 (1995).
\end{abstract}

Simian virus 40 (SV40) large $\mathrm{T}$ antigen ( $\mathrm{T}-\mathrm{ag}$ ) is known to cause extensive peripheral neuropathy (Messing et al., 1985 ) and to interfere with myelin formation and $P_{0}$ expression in vitro (Tennekoon et al., 1987; Bharucha et al., 1994). Also, in transgenic animals constitutively expressing the JC virus early region (which encodes the JC virus $\mathrm{T}$ antigens), dysmyelination occurs and myelin basic protein (MBP) mRNA, but not the protein itself, is expressed (Trapp et al., 1988). Recently, immortalized oligodendroglial cell lines have been prepared using the SV40 T-ag as the immortalizing oncogene, and in some cases it has been noted that these cell lines express MBP mRNAs but not the proteins (Jensen et al., 1993; Verity et al., 1993). These findings have led to the notion that viral $\mathrm{T}$ antigens might interfere with translation of the MBP mRNA.
Although most SV-40 T-ag has been observed to be localized within cell nuclei of infected cells, $\sim 5-10 \%$ has been observed in the cytoplasm (Michel and Schwyzer, 1982), which might be available to interfere with translation of certain mRNAs. Consistent with this notion is the finding that cytoplasmic SV40 T-ag appears to be bound to ribonucleoprotein structures, including polyribosomes (Michel and Schwyzer, 1982; Schwyzer et al., 1983). However, more recent data have shown that the SV40 T-ag possesses helicase activity, which might be expected to increase, rather than decrease, translational efficiency by decreasing the secondary structure of the mRNA (Scheffner et al., 1989). Thus, it is unclear whether or not T-ag interferes with translation of mRNAs.

In this report we have investigated directly the effects of SV40 T-ag on the translation of MBP mRNA. It appears that the SV40 T-ag does not inhibit translation of MBP mRNAs in transfected cells or in cell-free extracts. Thus, some other explanation must exist for the translational inhibition of MBP protein expression in SV40 immortalized oligodendrocyte cell lines.

\section{MATERIALS AND METHODS}

\section{Cell lines}

The mouse immortalized oligodendrocyte cell line N20.1 was engineered in our laboratory (Verity et al., 1993). COS1 cells (ATTC CRL 1650) and NIH 3T3 cells (ATCC CRL 6442) were obtained from the American Type Culture Collection. Cells were grown as described by Foster et al. (1993) at either $34(\mathrm{~N} 20.1)$ or $37^{\circ} \mathrm{C}(\mathrm{COS}-1$ and $\mathrm{NIH} 3 \mathrm{~T} 3$ cells).

Resubmitted manuscript received October 24, 1994; accepted October 28, 1994.

Address correspondence and reprint requests to Dr. A. T. Campagnoni at Developmental Biology Group, Mental Retardation Research Center, UCLA School of Medicine, 760 Westwood Plaza, Los Angeles, CA 90024, U.S.A.

Abbreviations used: MBP, myelin basic protein; SV40, simian virus 40; T-ag, large $\mathrm{T}$ antigen. 


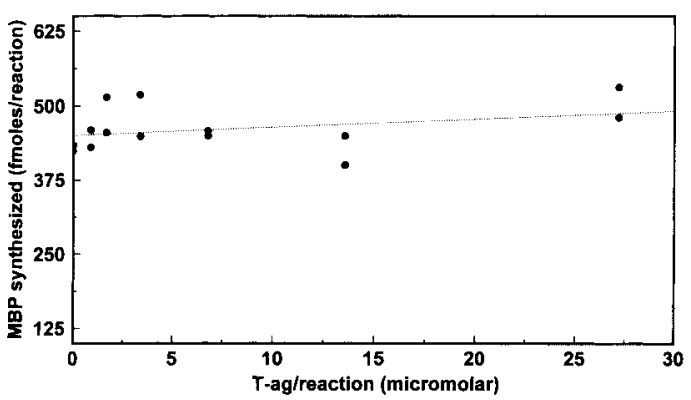

FIG. 1. Cell-free translation of 14-kDa MBP cRNA in the presence of varying concentrations of SV4O T-ag in rabbit reticulocyte lysates. MBP mRNA (0.1 pmol) was used in each incubation. The newly synthesized ${ }^{35} \mathrm{~S}-\mathrm{MBP}$ was immunoprecipitated, and the radioactivity was measured by scintillation counting. The horizontal axis presents the concentration of SV40 T-ag present in the incubation, and the vertical axis represents the femtomoles of immunoreactive MBP synthesized per reaction. Each point represents the average of two incubations, assayed in duplicate, at each concentration. There was no significant effect of T-ag on the translation of the MBP CRNA.

\section{SV40 T-ag preparation}

Sf 9 cells were infected with a baculovirus expression vector containing $\mathrm{T}$-ag. The protein was purified by the method of Lanford $(1988)$ and stored at $-20^{\circ} \mathrm{C}$.

\section{CRNA production and cell-free translations}

These were performed by techniques described previously (Ueno et al., 1994).

Permanent transfections of $\mathbf{N 2 0 . 1}$ and COS-1 cells

A full-length mouse 14-kDa MBP cDNA (Newman et al., 1987) was cloned into the EcoRI site of the eukaryotic expression vector pBabe-Puro (Morgenstern and Land, 1990). The construct was transfected into the N20.1 and COS- 1 cells by the calcium phosphate procedure of Chen and Okayama (1987). Transfections $(2.2 \mu \mathrm{g}$ of construct/ $\mathrm{ml}$ of medium) were carried out on 60 -mm-diameter plastic dishes containing either $5 \times 10^{5} \mathrm{~N} 20.1$ cells or $10^{5} \mathrm{COS}-1$ cells. The plates were incubated at $34^{\circ} \mathrm{C}$ under $3 \% \mathrm{CO}_{2}$ for $39 \mathrm{~h}$ with one medium change. The cells were trypsinized and passaged in the same medium. After $24 \mathrm{~h}$, the cells were changed to a standard medium containing $2.5 \mu \mathrm{g} / \mathrm{ml}$ of puromycin and maintained for 3-4 weeks. Individual colonies were then removed and examined for MBP expression by immunoblot analysis.

\section{Immunoblots}

Cell extracts were electrophoresed in either 15 (for MBP detection) or $7.5 \%$ (for SV40 T-ag detection) acrylamide gels in a vertical slab gel electrophoresis system as described previously (Ueno et al., 1994) and then electrophoretically transferred to nitrocellulose. One hundred micrograms of protein was applied to each lane. Electrochemiluminescence detection of the antibody-antigen complexes was performed according to the manufacturer's instructions (ECL system; Amersham). MBP was detected with a rabbit polyclonal antibody (Reidl et al., 1981) at a 1:10,000 dilution, and the SV40 T-ag was detected with a commercial monoclonal antibody (ATTC clone pAb101; Pharmingen) at a 1:250 dilution.

\section{RESULTS}

\section{Effect of SV40 T-ag on cell-free translation of MBP cRNAs}

It has been suggested that the inability of oligodendrocyte cell lines transformed with SV40 T-ag to express MBP, yet express the MBP mRNAs, could be due to interference with translation of the MBP mRNAs by T-ag (Jensen et al., 1993 ). To test this directly, synthetic, capped 14-kDa MBP cRNA was produced in vitro and translated in the presence of several concentrations of purified SV40 T-ag (Fig. 1). SV40 T-ag has been shown to affect the transcription of genes linearly up to a molar ratio equal to 25 times the concentration of the promoter (Rio et al., 1980). If the same molar ratio of T-ag known to inhibit transcription also inhibited translation, then $1 \mu M \mathrm{~T}$-ag should have been sufficient to inhibit translation based on the moles of MBP cRNA added to the cell-free translations. Concentrations of $\mathrm{T}$-ag up to $30 \mu M$ did not inhibit MBP mRNA translation in the reticulocyte lysates.

\section{Transfection of the MBP gene into SV40 \\ T-ag-expressing cell lines}

To examine the effect of SV40 T-ag on MBP gene expression in intact cells, we stably transfected mouse oligodendrocyte N20.1 cells and monkey kidney COS-1 cells with a full-length cDNA encoding the 14-kDa mouse MBP. The N20.1 cell line had been prepared by immortalizing mouse oligodendrocytes with a temperature-sensitive SV40 T-ag (Frederiksen et al., 1988), and the line has been observed to express MBP mRNAs but not the corresponding proteins by western blot analysis (Verity et al., 1993). The COS-1 cell line is a cell line transformed with SV40 T-ag. Both lines express high levels of SV40 T-ag, so they are suitable for examining the effect of endogenous $\mathrm{T}$-ag on the expression of the transfected MBP gene.

Figure 2 shows an MBP immunoblot of a COS- 1 cell line that has been stably transfected with the 14-kDa MBP construct (lane 2) and a nontransfected control (lane 1). Both the N20.1 and COS-1 cell lines expressed SV40 T-ag (see Fig. 3B, lanes 1 and 5), indicating that expression of T-ag did not inhibit translation of the transfected mRNAs. In similar experiments with the N20.I cell line, three stably transfected N20.1 cell lines were examined by MBP immunoblot analysis (Fig. 3A, lanes 2-4), all of which expressed MBP protein. Both nontransfected (Fig. 3B, lane 1) and transfected (Fig. 3B, lanes 2-4) N20.1 cells expressed high levels of SV40 T-ag.

\section{DISCUSSION}

The SV40 T-ag is a multifunctional nuclear phosphoprotein that shares the ability both to activate and to repress

FIG. 2. Expression of MBP in transfected COS-1 cells, shown in an MBP immunoblot of cell extracts of COS-1 cells either untransfected (lane 1) or stably transfected (lane 2) with a 14$\mathrm{kDa} \mathrm{MBP}$ cDNA. Lane 3 is an adult mouse brain extract control to serve as a marker for the MBP isoforms. Expression of the MBP could be detected in the transfected COS cells even though they express SV40 T-ag (see Fig. 3, lane 5).

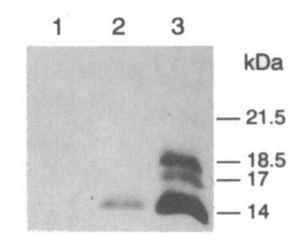




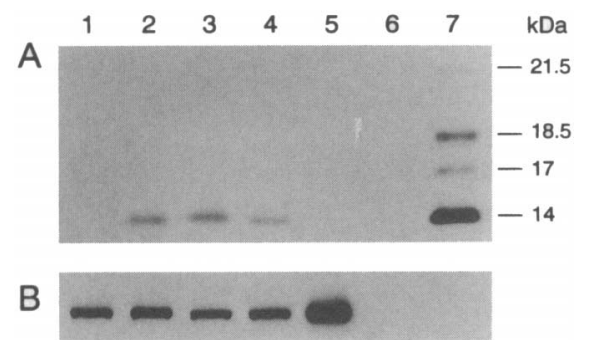

FIG. 3. Expression of MBP in transfected N20.1 cells. Immunoblots were prepared using (A) anti-MBP and (B) anti-SV40 Tag: lane 1, untransfected N20.1 cells; lanes 2-4, three separate N20.1 cell lines stably transfected with MBP CDNA; lane 5, untransfected COS-1 cells; lane 6, NIH 3T3 cells; and lane 7, adult mouse brain control. Expression of the 14-kDa MBP could be detected in the stably transfected N20.1 cells, and the SV40 Tag could be detected in the N20.1 and COS-1 cells.

gene transcription in mammalian cells (Tjian, 1981). It is also a useful viral product with which to induce immortalization as well as neoplastic transformation of mammalian cells (Chou and Martin, 1975). The mechanisms by which SV40 $\mathrm{T}$-ag functions remain unclear but may be related to the ability of $\mathrm{T}$ antigen to form a tight complex with nuclear phosphoprotein pS3 (Crawford et al., 1981), to bind to double-stranded DNAs (Clark et al., 1983), and/or to its protein phosphorylating activity (Griffin et al., 1979). There is little information about its action at the translational level.

It has been demonstrated that myelin-specific genes expressed in Schwann cells are susceptible to the effects of T-ag in vitro (Tennekoon et al., 1987; Bharucha et al., 1994). Two SV40 T-ag immortalized oligodendrocyte cell lines have been developed recently that express the MBP mRNA but not the corresponding proteins. Verity et al. (1993) produced an immortalized cell line by retroviral infection of cultured oligodendrocyte with a temperature-sensitive SV40 T-ag. Jensen et al. (1993) produced a similar cell line from tumors induced in transgenic mice with a transgene containing the MBP promoter to drive the expression of the wild-type SV40 T-ag in oligodendrocytes. These authors suggested that the SV40 T-ag might suppress expression of MBP protein posttranscriptionally, perhaps through selective inhibition of MBP mRNA translation.

The evidence obtained in this study suggests that this is probably not the case. The fact that translation of the 14$\mathrm{kDa}$ MBP cRNA is not inhibited by very large amounts ( 30 $\mu M$ ) of T-ag is direct evidence of this, and the finding that MBP protein is produced from a transfected gene in cell lines like COS- 1 that express T-ag abundantly supports this notion further. In the untransfected $\mathrm{N} 20.1$ cell line, at $39^{\circ} \mathrm{C}$ we cannot detect any greater levels of MBP by western blot analysis than at $34^{\circ} \mathrm{C}$, even though the levels of $\mathrm{T}$-ag are lower and it is inactivated (Verity et al., 1993). Similarly, in the N20.1 cell lines that were permanently transfected with the 14-kDa MBP cDNA we did not observe greater expression of the MBP by western blot analysis when the cells were grown at $39^{\circ} \mathrm{C}$ (data not shown).

In these studies we have focused on the 14-kDa MBP cRNAs because this mRNA is not produced by the N20.1 line and it would permit us to demonstrate unambiguously expression of the transfected gene in this cell line. The formal possibility exists that, owing to additional sequences within the coding region, the overall structure of the mRNAs for the larger MBP isoforms is sufficiently different from that of the mRNA encoding the 14-kDa MBP that the T-ag only inhibits the translation of those isoforms. This seems unlikely, however, because most regulatory elements in mRNAs exist in the untranslated regions, and also, as noted earlier, the larger MBPs are not expressed at $39^{\circ} \mathrm{C}$ in N20.1 cells when the T-ag is inactivated. We have not performed a quantitative analysis in the transfected cell lines to determine if a threshold level exists above which T-ag acts. However, the cell-free reticulocyte lysate data show no effect of T-ag on translation of the MBP mRNAs even at 30 times the concentration required to inhibit transcription.

In summary, our data do not support the notion that T-ag inhibits expression of the MBP gene at the translational level. Our results suggest that in the immortalized cell lines recently generated (Jensen et al., 1993; Verity et al., 1993), some other mechanism probably exists for the inhibition of MBP expression.

Acknowledgment: This work was supported, in part, by NIH grants NS 23022, NS 23322, and HD 25831 and grant RG 2233A1 from the National Multiple Sclerosis Society to A.T.C. and NIH grant NS 21700 to G.I.T.

\section{REFERENCES}

Bharucha V. A., Peden K. W. C., and Tennekoon G. I. (1994) SV40 large $T$ antigen with $c-j u n$ down-regulates myelin $\mathrm{P}_{0}$ gene expression: a mechanism for papovaviral $\mathrm{T}$ antigen-mediated demyelinating diseases. Neuron 12, 627-637.

Chen C. and Okayama H. (1987) High-efficiency transformation of mammalian cells by plasmid DNA. Mol. Cell. Biol. 7, 27452752.

Chou J. and Martin R. (1975) DNA infectivity and the induction of DNA synthesis with temperature-sensitive mutants of simian virus 40. J. Virol. 15, 145-155.

Clark R., Peden K., Pipas J., Nathans D., and Tjian R. (1983) Biochemical activities of $\mathrm{T}$ antigen proteins encoded by simian virus 40A gene deletion mutants. Mol. Cell. Biol. 3, 220-228.

Crawford L., Pim D., Gurney E., Goodfellow P., and Taylor-Papadimitriou J. (1981) Detection of a common feature in several human tumor cell lines-a 53,000 dalton protein. Proc. Natl. Acad. Sci. USA 78, 41-45.

Foster L. M., Phan T., Verity A. N., Bredesen D., and Campagnoni A. T. (1993) Generation and analysis of normal and shiverer temperature-sensitive immortalized cell lines exhibiting phenotypic characteristics of oligodendrocytes at several stages of differentiation. Dev. Neurosci. 15, 100-109.

Frederiksen K., Jat P. S., Valtz N., Levy D., and McKay R. (1988) Immortalization of precursor cells from the mammalian CNS. Neuron 1, 439-448.

Griffin J., Spangler G., and Livingstone D. (1979) Protein kinase activity associated with simian virus 40 tumor antigen. Proc. Natl. Acad. Sci. USA 76, 2610-2614.

Jensen N. A., Smith G. M., Shine H. D., Garvey J. S., and Hood L. (1993) Distinct hypomyelinated phenotypes in MBP SV40 large T transgenic mice. J. Neurosci. Res. 34, 257-264.

Lanford R. E. (1988) Expression of simian virus 40T antigen in insect cells using a baculovirus expression vector. Virology 167 , $72-81$.

Messing A., Chen H. Y., Palmiter R. D., and Brinster R. L. (1985) Peripheral neuropathies, hepatocellular carcinomas and islet cell adenomas in transgenic mice. Nature 316, 461-463.

Michel M. R. and Schwyzer M. (1982) Messenger ribonucleoproteins of cells infected by simian virus 40 contain large $T$ antigen. Eur. J. Biochem. 129, 25-32.

Mitchell P. J., Wang C., and Tjian R. (1987) Positive and negative regulation of transcription in vitro: enhancer-binding protein AP-2 is inhibited by SV40 T antigen. Cell 50, 847-861. 
Morgenstern J. P. and Land H. (1990) Advanced mammalian gene transfer: high titre retroviral vectors with multiple drug selection markers and a complementary helper-free packaging cell line. Nucleic Acids Res. 18, 3587-3596.

Newman S., Kitamura K., and Campagnoni A. T. (1987) Identification of a cDNA coding for a fifth form of myelin basic protein in mouse. Proc. Natl. Acad. Sci. USA 84, 886-890.

Reidl L. S., Campagnoni C. W., and Campagnoni A. T. (1981) Preparation and properties of an immunosorbent column specific for the myelin basic protein. $J$. Neurochem. 37, 373-380.

Rio D., Robbins A., Myers R., and Tjian R. (1980) Regulation of simian virus 40 early transcription in vitro by a purified tumor antigen. Proc. Natl. Acad. Sci. USA 77, 5706-5710.

Scheffner M., Knippers R., and Stahl H. (1989) RNA unwinding activity of SV40 large $\mathrm{T}$ antigen. Cell 57, 955-963.

Schwyzer M., Taj Y., Studer E., and Michel M. R. (1983) Binding sites for monoclonal antibodies and for mRNPs on SV40 large T-antigen determined with a cleavage map. Eur. J. Biochem. 137, 303-309.
Tennekoon G. I., Yoshino J., Peden K. W. C., Bigbee J., Rutkowski J. L., Kishimoto Y., DeVries G. H., and McKhann G. M. ( 1987) Transfection of neonatal rat Schwann cells with SV40 large T antigen gene under control of the metallothionein promoter. $J$. Cell Biol. 105, 2315-2325.

Tjian R. (1981) T antigen binding and the control of SV40 gene expression. Cell 26, 1-2.

Trapp B. D., Small J. A., Pulley M., Khoury G., and Scangos G. A. (1988) Dysmyelination in transgenic mice containing JC virus early region. Ann. Neurol. 23, 38-48.

Ueno S., Handley V. W., Byravan S., and Campagnoni A. T. (1994) Structural features of myelin basic protein mRNAs influence their translational efficiencies. J. Neurochem. 62, 1254-1259.

Verity A. N., Bredesen D., Vonderscher C., Handley V. W., and Campagnoni A. T. (1993) Expression of myelin protein genes and other myelin components in an oligodendrocytic cell line conditionally immortalized with a temperature-sensitive retrovirus. J. Neurochem. 60, 577-587. 